\title{
Age-metallicity-velocity relation of stars as seen by RAVE
}

\author{
Jennifer Wojno ${ }^{1}$, Georges Kordopatis ${ }^{1}$, Matthias Steinmetz ${ }^{1}$, \\ Gal Matijevič $\check{c}^{1}$, Paul J. McMillan ${ }^{2}$, the RAVE Collaboration \\ ${ }^{1}$ Leibniz-Institut für Astrophysik Potsdam (AIP), \\ An der Sternwarte 16, 14482 Potsdam, Germany \\ ${ }^{2}$ Lund Observatory, Lund University, Department of Astronomy and Theoretical Physics, \\ Box 43, SE-22100 Lund, Sweden
}

\begin{abstract}
Throughout the past decade, significant advances have been made in the size and scope of large-scale spectroscopic surveys, allowing for the opportunity to study in-depth the formation history of the Milky Way. Using the fourth data release of the RAdial Velocity Experiment (RAVE), we study the age-metallicity-velocity space of $\sim 100,000$ FGK stars in the extended solar neighborhood in order to explore evolutionary processes. Combining these three parameters, we better constrain our understanding of these interconnected, fundamental processes.
\end{abstract}

Keywords. stars: ages, stars: abundances, stars: kinematics and dynamics, Galaxy: formation

\section{Introduction}

Stellar ages are a crucial component of exploring the evolutionary history of the Milky Way. However, there are a number of obstacles facing reliable age estimations, as stellar ages are degenerate with a number of other factors in modern evolutionary models (e.g. metallicity, extinction, evolutionary tracks, see Soderblom 2010). Distances and age estimates for field stars were included in the fourth data release of RAVE (DR4) (Kordopatis et al. 2013). Binney et al. (2014) describe the method used to derive these parameters, and provide a comparison of the determined distances for a sample of stars with parallaxes measured by Hipparcos. We assess the validity of these ages, by generating a mock RAVE catalog using the stellar population synthesis code Galaxia (Sharma et al. 2011). This mock catalog is compared to a sample of RAVE stars which have been selected using a number of quality criteria (i.e., signal-to-noise ratio of the spectra, pipeline convergence, single stars only).

\section{Trend Computations and Interpretation}

Age-Metallicity Relation. First, we investigate the age-metallicity relation (AMR) for our RAVE sample. The distribution was modeled using a simple piecewise linear function, and fit to the data taking into account the uncertainties in both age and metallicity. The resulting fits are shown in Fig. 1. For the RAVE sample, the AMR for stars younger than $\sim 7$ Gyr is flat, while there is a clear correlation for older stars. The 'knee' of the distribution occurs at $\sim 6.5 \mathrm{Gyr}$, however, it is important to note that the position of this knee is heavily influenced by biases introduced by the age determination method. These trends are also seen in the mock RAVE catalog, and agree with trends shown for similar samples of local solar neighborhood stars $(\mathrm{d}<200 \mathrm{pc})$, such as in Haywood et al. (2013) and Bensby et al. (2014). 

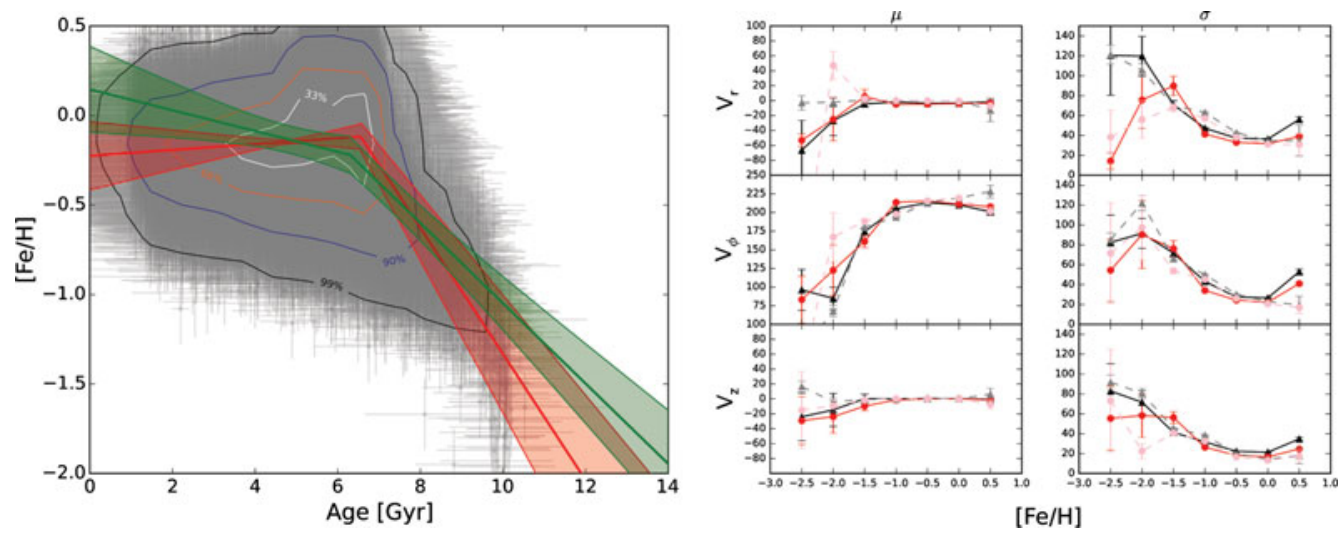

Figure 1. Left: Age v. [Fe/H] distribution for a sample of RAVE stars (grey). The resulting fit and uncertainties are shown in red. This same model was fit to the mock RAVE catalog generated by the Galaxia code, and the resulting fit is shown in green.

Right: Mean velocities (left) and velocity dispersion (right) for each velocity component as a function of metallicity. RAVE trends are indicated by the solid lines, and the mock RAVE catalog by dashed lines. In each panel, dwarfs are indicated by red circles, and giants by black triangles.

Velocity-Metallicity Relation. In order to ensure that the sample consists of only bound stars, we limited the selection to those stars with $V_{t o t}<V_{e s c}$, where we adopted the lower limit for the Galactic escape speed determined in Piffl et al. (2014) of $492 \mathrm{~km} \mathrm{~s}^{-1}$. The resulting mean velocities and velocity dispersions are shown in Fig. 1. The velocity dispersions decrease with increasing metallicity, although the most metal-poor stars deviate greatly from the general trend. However, these metallicity bins are sparsely populated, and likely suffer from small number statistics. In each of the components, there is also a distinct increase in velocity dispersion for the most metal-rich stars.

\section{Conclusions}

From the comparisons shown in Fig. 1, it is clear that the age estimates in RAVE DR4 are useable for future analysis. We recover physical phenomena (such as asymmetric drift) present in the velocity-metallicity space of the RAVE data with the mock catalog, as well as match the trends in the velocity dispersions as a function of metallicity with those as a function of age, indicating a correlation. For future studies, the RAVE sample will be divided into age bins, in order to better explore the parameter space for a given mono-age population, where these age estimates may be better constrained by including photometric measurements in addition to the stellar parameters derived from spectra (Wojno et al., in preparation). Furthermore, the procedure outlined in Binney et al. (2014) will be updated and improved; in particular, the priors used in determining ages, which may influence any biases in the currently observed trends, will be relaxed.

\section{References}

Bensby, T., Feltzing, S., \& Oey, M. S. 2014, A\&A, 562, 71

Binney, J., Burnett, B., Kordopatis, G., et al. 2014, MNRAS, 437, 351

Haywood, M., Di Matteo, P., Lehnert, M., Katz, D., \& Gómez, A. 2013, A\&\&A, 560, 109

Kordopatis, G., Gilmore, G., \& Steinmetz, M. et al. 2013, AJ, 146, 134

Piffl, T., Scannapieco, C., Binney, J., et al. 2014, A\& $A, 562,91$

Sharma, S., Bland-Hawthorn, J., Johnston, K. V., \& Binney, J. 2011, ApJ, 730, 3

Soderblom, D. R. 2010, ARAA, 48, 581 